\title{
PROBABLE CREUTZFELDT JAKOB DISEASE: CASE REPORT
}

\author{
${ }^{1}$ Yanes Brum Bello, ${ }^{2}$ Alexandre Cossenza Pettezzoni De Almeida \\ ${ }^{2}$ Daniela Mendonca Sueth, ${ }^{2}$ Eduardo Carvalho Belizario, \\ ${ }^{3}$ Fabiola Sampaio Brandao, ${ }^{4}$ Marco Araujo Leite, \\ ${ }^{4}$ Marco Orsini and ${ }^{1}$ Antonio Marcos Da Silva Catharino \\ ${ }^{1}$ Department of Neurology, Iguacu University, Rio de Janeiro, Brazil \\ ${ }^{2}$ Department of ICU Surgery Clinic Santa Barbara, Rio de Janeiro, Brazil \\ ${ }^{3}$ Department of Rheumatology Municipal Hospital Governador Valadares, Minas Gerais, Brazil \\ ${ }^{4}$ Department of Neurology Federal, University Fluminense, Rio de Janeiro, Brazil
}

Received 2013-09-08; Received 2014-03-09; Accepted 2014-03-14

\begin{abstract}
Prion diseases are associated with the accumulation of an abnormal isoformof cellular prion protein $\left(\mathrm{PrP}^{\mathrm{Sc}}\right)$, which is the principal constituent of prions. Prions replicate in lymphoreticular tissues before neuroinvasion, suggesting that lymphoreticular biopsy samples may allow early diagnosis by detection of $\operatorname{PrP}^{\mathrm{Sc}}$. Creutzfeldt-Jakob Disease (CJD) is the most frequent prion disease in humans. Clinical diagnosis of sporadic (CJD) is based on the evaluation of rapidly progressive dementia, ataxia, myoclonus, changes on the electroencephalogram and other neurological signs. We report a problable (CJD) case in Santa Barbara surgical center, Rio de Janeiro, Brazil. From our clinical case, we decided to do a brief review about (CJD) researching at Medline and Pubmed, using terms Creutzfeldt-Jakob disease and Prion diseases. A definite diagnosis, however, is confined to cases that have been evaluated neuropathlogically or by equivalent diagnostic techniques. The range in clinical expression of the disease is better appreciated and the existence of "atypical" cases of CJD is increasingly recognized. The clinical characteristics, laboratory findings, differential diagnosis, mechanisms of transmission and the actual therapeutic approach are discussed.
\end{abstract}

Keywords: Creutzfeldt-Jakob Disease, Prion Diseases, Diagnosis

\section{INTRODUCTION}

Creutzfeldt-Jakob Disease (CJD) is a rare, degenerative, invariably fatal brain disorder. Typically, onset of symptoms occurs about age 60 and about 90 percent of individuals die within 1 year. In the early stages of disease, patients may have memory complains, behavioral changes, ataxia and visual disturbances. As the illness progresses, mental deterioration becomes pronounced and involuntary movements, blindness, weakness of extremities and coma may occur. CJD belongs to a family of human and animal diseases known as the Transmissible Spongiform Encephalopathies (TSEs).
Spongiform refers to the characteristic appearance of infected brains, which become filled with holes until they resemble sponges under a microscope. CJD is the most common of the known human TSEs (NASS, 2012; Sikorska et al., 2012).

\subsection{Case Report}

Patient 68, female, of Portuguese descent, previously healthy, was admitted to the ICU of the Santa Barbara Surgical Center in Rio de Janeiro-RJ, with signs of urinary sepsis. Family members reported that the patient had suffered from tremors in the upper limbs for 5 months, progressing to ataxia after a few Corresponding Author: Yanes Brum Bello, Department of Neurology, Iguacu University, Rio de Janeiro, Brazil 
days. After 2 months the patient was confined to bed due to the severity of the ataxia. During this period the patient developed apathy, becoming slightly communicative and having little interaction with family members. A research into other services was initiated, being diagnosed with cerebellar syndrome in the four members by her last neurologist and associated to hyperreflexia, prompting an initial investigation to Machado-Joseph disease. The resonance of the skull showed the onset of cerebellar atrophy, with enlargement of the 4 th ventricle.

On admission to the ICU, the patient presented signs of sepsis, urinary Glasgow 9 (eye opening 4, a verbal response 1 , motor response 4), being observed intense cogwheel rigidity, tremor at fine rest and symmetrical in the four members, more intense myoclonus on the left shoulder that was accentuated with passive stimuli, nystagmus polysynthetic and inexhaustible and akinetic mutism. The tendon reflexes had slowed to $(1+/ 5+)$ with bilateral cutaneous plantar flexion. After the initial evaluation, lumbar puncture was performed for collecting cerebrospinal fluid, with an initial pressure of $14 \mathrm{cmH}$ 20 , clear and colorless, leukocyte 1 , erythrocytes 0 , proteins $22 \mathrm{mg} \mathrm{dL}^{-1}$ and glucose $55 \mathrm{mg} \mathrm{dL}^{-1}$. The cultures (bacteria, fungi and AFB), VDRL, Gram, Nanquim, Latex and PCR for viruses, fungi and bacteria were negative (Neurocysticercosis, Neurolues, Neurotoxoplasmosis, Herpes Simplex, cytomegalovirus, Varicella-zoster virus, HIV, HTLV 1 and 2 as with research for neoplastic cells.
After these results we chose to perform a new MRI of the skull with a 3 tesla machine in which showed an increased diffuse signal on $\mathrm{T} 2$ and a bilateral FLAIR in the caudate and putamen nucleus (Fig. 1), with restricted of the diffusion without significant capture of the contrast. After these observations, associated to the clinical picture of sub-acute cognitive decline, myoclonus and extra-pyramidal and cerebellar signs, we requested the 14-3-3 protein be tested in CSF in which showed positive. The search for cellular prion gene polymorphism was negative.

There was no evidence of autoimmune diseases or rheumatic (anti-TPO rheumatoid factor, ANCA, ANA, anti-Ro, anti-La, anti-SM, anti-DNA double helix, Anticardiolipin negative), paraneoplasias (chest CT, abdomen and pelvis normal and test for alpha fetoprotein, CA 125, anti-Yo, anti-Hu and normal protein serum electrophoresis), endocrine-metabolic (normal TSH, FT4, PTH, vitamin B12, folic acid, copper serum, ceruloplasmin, iron, ferritin, transferrin, electrolytes, homocysteine, renal function and liver function) or vascular. The initial electroencephalogram was normal, but we observed in subsequent examinations the appearance of diffused slow stroke (onset of diffused theta waves).

Based on the clinical state of the patient it was classified, according to the classification of Prion diseases of the world health organization which is also followed by the ministry of health of Brazil, as probable Creutzfeldt Jakob Disease in a sporadic form ${ }^{1}$. After three months in the ICU, the patient progressed to death with no family consent to carry out autopsies.

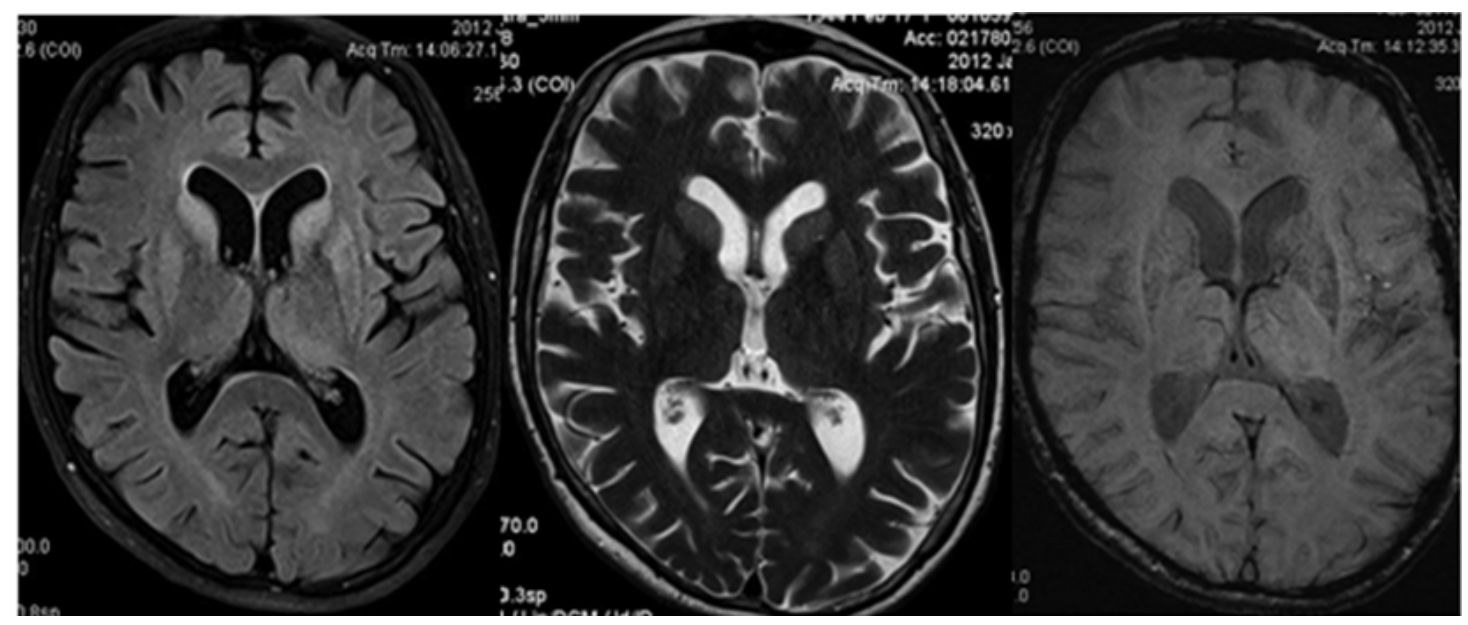

Fig. 1. Increased signal on $T 2$ and a bilateral FLAIR in the caudate and putamen nucleus, with restricted of the diffusion. 


\section{DISCUSSION}

Creutzfeldt Jakob Disease (CJD) is a neurodegenerative disease and the most common form of prion disease in humans. There are four types of the Creutzfeldt Jacob Disease: The classical (sporadic, genetic or familial, iatrogenic) and the variant form. The term "classic CJD" is used to describe all forms of TSEs that affect humans, excluding the variant form. This group can include: sporadic CJD, genetic CJD, CJD iatrogenic, Gerstmanntraussler-Scheinker disease, fatal familial Insomnia and the Kuru (Sikorska et al., 2012).

CJD is a rare neurological disorder with a prevalence of approximately 1 case per 1 million inhabitants, or approximately 1/10000 cases of Alzheimer's disease. Recently CJD caused alarm throughout Europe due to an epidemic of bovine spongiform encephalopathy (the "crazy cow" disease), this form being classified as variant CJD (Paterson et al., 2012). The variant CJD is more common in young adults, in contrast to the sporadic form that occurs commonly in patients over 50 years of age.

In the variant type CJD the demonstration of hyper intensity in the pulvinar nucleus of the thalamus by brain magnetic resonance, has high accuracy for the diagnosis of the variant type of CJD and the FLAIR is more sensitive than other sequences. Patients with high hyper intense basal ganglia on T2 are more likely to present rapid progressive dementia in an initial stage and a shorter time span of the illness (average of 6.7 and 8.6 months). Depression and sensory disturbances are more frequent among cases without increased signs (Paterson et al., 2012).

Several diseases can mimic CJD, including infections of the central nervous system (Whipple's disease, viral encephalitis), primary and secondary dementias and the autoimmune encephalopathy, especially Hashimoto Encephalopathy, paraneoplasias and metabolic disorders ${ }^{6}$. There were no other findings in our patient suggesting any of these disorders, beyond the screening previously cited, negative results were obtained (McKeon et al., 2010).

\section{CONCLUSION}

The clinical diagnosis of the CJD can be best supported by the 14-3-3 test and cortical signal increase on MRI. MRI plays an important role in differential diagnosis and should be performed early during the disease course.

\section{REFERENCES}

McKeon, A., V.A. Lennon and S.J. Pittock, 2010. Immunotherapy-responsive dementias and encephalopathies. Continuum Lifelong Learn., 16: 80-101. DOI: 10.1212/01.CON.0000368213.63964.34, PMID: 22810282

NASS, 2012. Technical notebook: Transmissible spongiform encephalopathy.

Paterson, R.W., C.C.T. Chae and A.L. Kuo, 2012. Differential diagnosis of Jakob-creutzfeldt disease. Arch. Neurol. 69: 1578-1582. DOI: 10.1001/archneurol.2013.79

Sikorska, B., R. Knight, J.W. Ironside and P.P. Liberski, 2012. Creutzfeldt-Jakob disease. Adv. Exp. Med. Biol., 724: 76-90. DOI: 10.1007/978-1-4614-06532_6 